\title{
Bifurcation Analysis for a Kind of Nonlinear Finance System with Delayed Feedback and Its Application to Control of Chaos
}

\author{
Rongyan Zhang \\ Department of Electronic Information Engineering, Huanghe Science and Technology College, \\ Henan, Zhengzhou 450063, China \\ Correspondence should be addressed to Rongyan Zhang, hebgydx2006@163.com
}

Received 14 October 2011; Revised 15 January 2012; Accepted 23 January 2012

Academic Editor: Chuanhou Gao

Copyright (c) 2012 Rongyan Zhang. This is an open access article distributed under the Creative Commons Attribution License, which permits unrestricted use, distribution, and reproduction in any medium, provided the original work is properly cited.

\begin{abstract}
A kind of nonlinear finance system with time-delayed feedback is considered. Firstly, by employing the polynomial theorem to analyze the distribution of the roots to the associate characteristic equation, the conditions of ensuring the existence of Hopf bifurcation are given. Secondly, by using the normal form theory and center manifold argument, we derive the explicit formulas determining the stability, direction, and other properties of bifurcating periodic solutions. Finally, we give several numerical simulations, which indicate that when the delay passes through certain critical values, chaotic oscillation is converted into a stable steady state or a stable periodic orbit.
\end{abstract}

\section{Introduction}

Since the chaotic phenomenon in economics was first found in 1985, great impact has been imposed on the prominent western economics at present, because the chaotic phenomenon occurring in the economic system means that the macroeconomic operation has in itself the inherent indefiniteness. Although the government can adopt such macrocontrol measures as the financial policies or the monetary policies to interfere, the effectiveness of the interference is very limited. The instability and complexity make the precise economic prediction greatly limited, and the reasonable prediction behavior has become complicated as well. In the fields of finance, stocks, and social economics, because of the interaction between nonlinear factors, with all kinds of economic problems being more and more complicated and with the evolution process from low dimensions to high dimensions, the diversity and complexity have manifested themselves in the internal structure of the system and there exists extremely 


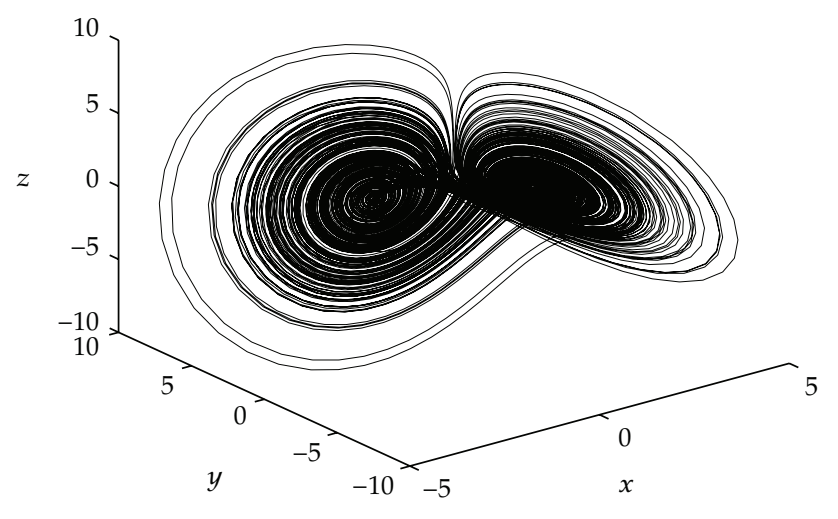

Figure 1: Strange attractor of finance system (1.1).

complicated phenomenon and external characteristics in such a kind of system. So it has become more and more important to study the control of the complicated continuous economic system and stabilize the instable periodic or stationary solutions, in order to make the precise economic prediction possible $[1,2]$.

Recent works $[1,2]$ have reported a dynamic model of finance, composed of three first-order differential equations. The model describes the time variations of three state variables: the interest rate $x$, the investment demand $y$, and the price index $z$. By choosing an appropriate coordinate system and setting appropriate dimensions for each state variable, $[1,2]$ offer the simplified finance system as

$$
\begin{gathered}
\dot{x}(t)=-a(x(t)+y(t)), \\
\dot{y}(t)=-y(t)-a x(t) z(t), \\
\dot{z}(t)=b+a x(t) y(t),
\end{gathered}
$$

which is chaotic when $a=1.69, b=4$ (see Figure 1 ).

Over the last years, $[3,4]$ studied impulsive control and state feedback control of the finance system (1.1). In this paper, we are interesting in delayed feedback control of the finance system (1.1). The effects of the time-delayed feedback on the finance system have long been investigated [5-8].

Recently, different techniques and methods have been proposed to achieve chaos control. The existing control methods can be classified, mainly, into two categories. The first one, developed by Ott et al. [9] is based on the invariant manifold structure of unstable orbits. It is theoretically well understood but difficult to apply to fast experimental systems. The second, proposed by Pyragas [10], uses time-delayed controlling forces. In contrast to the former one, it is simple and convenient method of controlling chaos in continuous dynamical system. Thus, we adopt the second one in the present paper. 
For predigesting the investigation, here we only put time delay on investment demand $y$. By adding a time-delayed force $K(y(t)-y(t-\tau))$ to the second equation of finance system (1.1), we obtain the following new system

$$
\begin{gathered}
\dot{x}(t)=-a(x(t)+y(t)), \\
\dot{y}(t)=-y(t)-a x(t) z(t)+K(y(t)-y(t-\tau)), \\
\dot{z}(t)=b+a x(t) y(t) .
\end{gathered}
$$

Here we assume that $\left(C_{1}\right) a, b, \tau \in(0, \infty)$ and $K \in R$. The time delay $\tau$ is taken as the bifurcation parameter and we show that when $\tau$ passes through some certain critical values, the equilibrium will lose its stability and hopf bifurcation will take place; by adjusting $K$ values, we achieve the purpose of chaos control. The research of this paper is a new investigation about the hopf bifurcation and chaos control on the finance system and has important theoretical and practical value.

\section{Stability of Steady States and Bifurcations of Periodic Solutions}

In this section, we investigate the effect of delay on the dynamic behavior of system (1.2). Obviously, when $\tau=0$, system (1.2) becomes the system (1.1). First, we introduce the following several lemmas in $[1,2]$ for T's system(1.1).

We know that under the assumption $\left(C_{1}\right)$, the system (1.1) has two equilibrium points:

$$
S_{1}=\left(\frac{\sqrt{a b}}{b},-\frac{\sqrt{a b}}{b}, \frac{1}{a}\right), \quad S_{2}=\left(-\frac{\sqrt{a b}}{b}, \frac{\sqrt{a b}}{b}, \frac{1}{a}\right) .
$$

The characteristic equation of the system $(1.1)$ at $S_{1}\left(S_{2}\right)$ is

$$
\lambda^{3}+(1+a) \lambda^{2}+a b \lambda+2 a^{2} b=0
$$

By analyzing the characteristic equation (2.2) and the Routh-Hurwitz criteria, we get the following.

Lemma 2.1. For $a<1$, the characteristic equation (2.2) has three eigenvalues with negative real parts, so two equilibrium points $S_{1}, S_{2}$ of the system (1.1) are asymptotic stable.

Lemma 2.2. For $a=1$, the characteristic equation (2.2) has a pair of purely imaginary eigenvalues $\lambda_{1,2}= \pm i \omega_{0}\left(\omega_{0}=\sqrt{b}\right)$ and a negative real eigenvalue $\lambda_{3}=-2$, and

$$
\dot{\lambda}(a=1)=\frac{5 b}{2 b+8}>0 .
$$

According to the hopf bifurcation theorem [11], a hopf bifurcation of the system (1.1) occurs at a =1.

Lemma 2.3. For $a>1$, the characteristic equation (2.2) has one negative real root and one pair of conjugate complex roots with positive real parts, so two equilibrium points $S_{1}, S_{2}$ of the system (1.1) are unstable. 
Clearly, the delayed feedback control system (1.2) has the same equilibria to the corresponding system (1.1). In this section, we analyze the effect of delay on the stability of these steady states. Due to the symmetry of $S_{1}$ and $S_{2}$, it is sufficient to analyze the stability of $S_{1}$. By the linear transform

$$
\begin{gathered}
x_{1}(t)=x(t)-\frac{\sqrt{a b}}{b}, \\
y_{1}(t)=y(t)+\frac{\sqrt{a b}}{b}, \\
z_{1}(t)=z(t)-\frac{1}{a},
\end{gathered}
$$

system (1.2) becomes

$$
\begin{gathered}
\dot{x}_{1}(t)=-a\left(x_{1}(t)+y_{1}(t)\right), \\
\dot{y}_{1}(t)=-x_{1}(t)-y_{1}(t)-\sqrt{a b} z_{1}(t)-a x_{1}(t) z_{1}(t)+K\left(y_{1}(t)-y_{1}(t-\tau)\right), \\
\dot{z}_{1}(t)=-\sqrt{a b} x_{1}(t)+\sqrt{a b} y_{1}(t)+a x_{1}(t) y_{1}(t) .
\end{gathered}
$$

It is easy to see that the origin $S_{0}(0,0,0)$ is the equilibrium of system (2.5). The associated characteristic equation of system $(2.5)$ at $S_{0}(0,0,0)$ is

$$
\operatorname{det}\left(\begin{array}{ccc}
\lambda+a & a & 0 \\
1 & \lambda+1-K+K e^{-\lambda \tau} & \sqrt{a b} \\
\sqrt{a b} & -\sqrt{a b} & \lambda
\end{array}\right)=0
$$

Expanding (2.6), we have

$$
\lambda^{3}+(1+a-K) \lambda^{2}+(a b-a K) \lambda+2 a^{2} b+K\left(\lambda^{2}+a \lambda\right) e^{-\lambda \tau}=0 .
$$

Thus, we need to study the distribution of the roots of the third-degree exponential polynomial equation:

$$
\lambda^{3}+a_{2} \lambda^{2}+a_{1} \lambda+a_{0}+\left(b_{2} \lambda^{2}+b_{1} \lambda+b_{0}\right) e^{-\lambda \tau}=0
$$

where $a_{i}, b_{i} \in R(i=0,1,2)$ and $\sum_{i=0}^{2} b_{i}^{2} \neq 0$. We first introduce the following simple result which was proved by Ruan and Wei [12] using Rouche's theorem.

Lemma 2.4. Consider the exponential polynomial

$$
\begin{aligned}
P( & \left.\lambda, e^{-\lambda \tau_{1}}, \ldots, e^{-\lambda \tau_{m}}\right) \\
= & \lambda^{n}+p_{1}^{(0)} \lambda^{n-1}+\cdots+p_{n-1}^{(0)} \lambda+p_{n}^{(0)}+\left[p_{1}^{(1)} \lambda^{n-1}+\cdots+p_{n-1}^{(1)} \lambda+p_{n}^{(1)}\right] e^{-\lambda \tau_{1}} \\
& +\cdots+\left[p_{1}^{(m)} \lambda^{n-1}+\cdots+p_{n-1}^{(m)} \lambda+p_{n}^{(m)}\right] e^{-\lambda \tau_{m}}
\end{aligned}
$$


where $\tau_{i} \geq 0(i=1,2, \ldots, m)$ and $p_{j}^{(i)}(i=0,1, \ldots, m, j=1,2, \ldots, n)$ are constants. As $\left(\tau_{1}, \tau_{2}, \ldots, \tau_{m}\right)$ vary, the sum of the order of the zeros of $P\left(\lambda, e^{-\lambda \tau_{1}}, \ldots, e^{-\lambda \tau_{m}}\right)$ on the open right half plane can change only if a zero appears on or crosses the imaginary axis.

Obviously, $i \omega(\omega>0)$ is a root of (2.8) if and only if $\omega$ satisfies

$$
-i \omega^{3}-a_{2} \omega^{2}+a_{1} \omega i+a_{0}+\left(-b_{2} \omega^{2}+b_{1} \omega i+b_{0}\right)(\cos \omega \tau-i \sin \omega \tau)=0
$$

Separating the real and imaginary parts, we have

$$
\begin{aligned}
& a_{2} \omega^{2}-a_{0}=\left(b_{0}-b_{2} \omega^{2}\right) \cos \omega \tau+b_{1} \omega \sin \omega \tau \\
& -\omega^{3}+a_{1} \omega=\left(b_{0}-b_{2} \omega^{2}\right) \sin \omega \tau-b_{1} \omega \cos \omega \tau
\end{aligned}
$$

which is equivalent to

$$
\omega^{6}+\left(a_{2}^{2}-b_{2}^{2}-2 a_{1}\right) \omega^{4}+\left(a_{1}^{2}-2 a_{0} a_{2}-b_{1}^{2}+2 b_{0} b_{2}\right) \omega^{2}+a_{0}^{2}-b_{0}^{2}=0 .
$$

Let $z=\omega^{2}$ and denote $p=a_{2}^{2}-b_{2}^{2}-2 a_{1}, q=a_{1}^{2}-2 a_{0} a_{2}-b_{1}^{2}+2 b_{0} b_{2}, r=a_{0}^{2}-b_{0}^{2}$, then (2.12) becomes

$$
z^{3}+p z^{2}+q z+r=0
$$

In the following, we need to seek conditions under which (2.12) has at least one positive root. Denote

$$
h(z)=z^{3}+p z^{2}+q z+r .
$$

Therefor, applying [13], we obtain the following lemma.

Lemma 2.5. For the polynomial equation (2.13), one has the following results.

(i) If $r<0$, then (2.13) has at least one positive root.

(ii) If $r \geq 0$ and $\Delta=p^{2}-3 q \leq 0$, then (2.13) has no positive roots.

(iii) If $r \geq 0$ and $\Delta=p^{2}-3 q>0$, then (2.13) has positive roots if and only if $z_{1}^{*}=(1 / 3)(-p+$ $\sqrt{\Delta})>0$ and $h\left(z_{1}^{*}\right) \leq 0$.

Suppose that (2.13) has positive roots. Without loss of generality, we assume that it has three positive roots, defined by $z_{1}, z_{2}$, and $z_{3}$, respectively. Then (2.12) has three positive roots:

$$
\omega_{1}=\sqrt{z_{1}}, \quad \omega_{2}=\sqrt{z_{2}}, \quad \omega_{3}=\sqrt{z_{3}} .
$$


From (2.11), we have

$$
\cos \omega \tau=\frac{b_{1} \omega^{2}\left(\omega^{2}-a_{1}\right)-\left(a_{2} \omega^{2}-a_{0}\right)\left(b_{2} \omega^{2}-b_{0}\right)}{\left(b_{2} \omega^{2}-b_{0}\right)^{2}+b_{1}^{2} \omega^{2}} .
$$

Thus, if we denote

$$
\tau_{k}^{(j)}=\frac{1}{\omega_{k}}\left[\cos ^{-1}\left(\frac{b_{1} \omega_{k}^{2}\left(\omega_{k}^{2}-a_{1}\right)-\left(a_{2} \omega_{k}^{2}-a_{0}\right)\left(b_{2} \omega_{k}^{2}-b_{0}\right)}{\left(b_{2} \omega_{k}^{2}-b_{0}\right)^{2}+b_{1}^{2} \omega_{k}^{2}}\right)+2 j \pi\right]
$$

where $k=1,2,3 ; j=0,1,2, \ldots$, then $\pm i \omega_{k}$ is a pair of purely imaginary roots of $(2.8)$ with $\tau_{k}^{(j)}$. Define

$$
\tau_{0}=\tau_{k_{0}}^{(0)}=\min _{k \in 1,2,3} \tau_{k}^{(0)}, \quad \omega_{0}=\omega_{k_{0}}
$$

Note that when $\tau=0$, (2.8) becomes

$$
\lambda^{3}+\left(a_{2}+b_{2}\right) \lambda^{2}+\left(a_{1}+b_{1}\right) \lambda+a_{0}+b_{0}=0 .
$$

Therefor, applying Lemmas 2.4 and 2.5 to (2.8), we get the following lemma.

Lemma 2.6. For (2.8), one has

(i) if $r \geq 0$ and $\Delta=p^{2}-3 q \leq 0$, then all roots with positive real parts of (2.8) have the same sum to those of the polynomial equation (2.19) for all $\tau \geq 0$.

(ii) if either $r<0$ or $r \geq 0, \Delta=p^{2}-3 q>0, z_{1}^{*}=(1 / 3)(-p+\sqrt{\Delta})>0$ and $h\left(z_{1}^{*}\right) \leq 0$, then all roots with positive real parts of (2.8) have the same sum to those of the polynomial equation (2.19) for $\tau \in\left[0, \tau_{0}\right)$.

Let

$$
\lambda(\tau)=\alpha(\tau)+i \omega(\tau)
$$

be the root of (2.8) near $\tau=\tau_{k}^{(j)}$ satisfying

$$
\alpha\left(\tau_{k}^{(j)}\right)=0, \quad \omega\left(\tau_{k}^{(j)}\right)=\omega_{k}
$$

Then by [13], we have the following transversality condition. 
Lemma 2.7. Suppose that $z_{k}=\omega_{k}^{2}$ and $h^{\prime}\left(z_{k}\right) \neq 0$. Then

$$
\frac{R \lambda\left(\tau_{k}^{(j)}\right)}{d \tau} \neq 0
$$

and $R \lambda\left(\tau_{k}^{(j)}\right) / d \tau$ and $h^{\prime}\left(z_{k}\right)$ have the same sign.

Now, we study the characteristic equation (2.7) of the system (2.5). Comparing (2.7) with (2.8), we know that

$$
a_{2}=1+a-K, \quad a_{1}=a b-a K, \quad a_{0}=2 a^{2} b, \quad b_{2}=K, \quad b_{1}=a K, \quad b_{0}=0 .
$$

Thus,

$$
\begin{gathered}
p=a_{2}^{2}-b_{2}^{2}-2 a_{1}=a^{2}+2 a+1-2 a b-2 K \\
q=a_{1}^{2}-2 a_{0} a_{2}-b_{1}^{2}+2 b_{0} b_{2}=a^{2} b^{2}+2 a^{2} b K-4 a^{2} b-4 a^{3} b, \\
r=a_{0}^{2}-b_{0}^{2}=4 a^{4} b^{2}>0
\end{gathered}
$$

and then we can compute

$$
\Delta=p^{2}-3 q, \quad h(z)=z^{3}+p z^{2}+q z+r, \quad z_{1}^{*}=\frac{1}{3}(-p+\sqrt{\Delta}) .
$$

When $\tau=0$, (2.7) becomes (2.2)

$$
\lambda^{3}+(1+a) \lambda^{2}+a b \lambda+2 a^{2} b=0
$$

Applying Lemmas 2.1, 2.2, 2.6, and 2.7 to (2.7), we have the following theorems.

Theorem 2.8. Let $\tau_{k}^{(j)}$ and $\tau_{0}$ be defined by (2.17) and (2.18). Suppose that conditions $\left(C_{1}\right)$ and $a<1$ hold.

(i) If $\Delta \leq 0$, then (2.7) had all roots with negative real parts for all $\tau \geq 0$, and the equilibrium $S_{1}$ (or $S_{2}$ ) of the system (1.2) is stable.

(ii) If $\Delta>0, z_{1}^{*}>0$ and $h\left(z_{1}^{*}\right) \leq 0$, (2.7) had all roots with negative real parts for $\tau \in\left[0, \tau_{0}\right)$, and the equilibrium $S_{1}$ (or $S_{2}$ ) of the system (1.2) is stable.

(iii) If the conditions of (ii) are satisfied, and $h^{\prime}\left(z_{k}\right) \neq 0$, then system (1.2) exhibits the Hopf bifurcation at the equilibrium $S_{1}\left(\right.$ or $\left.S_{2}\right)$ for $\tau=\tau_{k}^{(j)}$.

Theorem 2.9. Let $\tau_{k}^{(j)}$ and $\tau_{0}$ are defined by (2.17) and (2.18). Suppose that conditions $\left(C_{1}\right)$ and $a>1$ hold.

(i) If $\Delta \leq 0$, then (2.7) had two roots with positive real parts for all $\tau \geq 0$, and the equilibrium $S_{1}\left(\right.$ or $\left.S_{2}\right)$ of the system (1.2) is unstable. 
(ii) If $\Delta>0, z_{1}^{*}>0$ and $h\left(z_{1}^{*}\right) \leq 0,(2.7)$ has two roots with positive real parts for $\tau \in\left[0, \tau_{0}\right)$, and the equilibrium $S_{1}$ (or $S_{2}$ ) of the system (1.2) is unstable.

(iii) If the conditions of (ii) are satisfied, and $h^{\prime}\left(z_{k}\right) \neq 0$, then system (1.2) exhibits the Hopf bifurcation at the equilibrium $S_{1}$ (or $S_{2}$ ) for $\tau=\tau_{k}^{(j)}$.

\section{Direction and Stability of the Hopf Bifurcation}

In the Section 2, we obtained some conditions which guarantee that the system (1.2) undergoes the Hopf bifurcation at a sequence values of $\tau$. In this section, we shall study the direction and stability of the Hopf bifurcation. The method we used is based on the normal form theory and the center manifold theorem introduced by Hassard et al. [14]. Throughout this section, we always assume that system (1.2) undergoes Hopf bifurcations at the steady state $\left(x_{*}, y_{*}, z_{*}\right)$ for $\tau=\tau_{k}$ and then $\pm i \omega_{k}$ is corresponding purely imaginary roots of the characteristic equation at the steady state $\left(x_{*}, y_{*}, z_{*}\right)$.

Letting $x_{1}=x-x_{*}, x_{2}=y-y_{*}, x_{3}=z-z_{*}, \bar{x}_{i}(t)=x_{i}(\tau t), \tau=\tau_{k}+\mu$ and dropping the bars for simplification of notations, system (1.2) is transformed into an FDE in $C=C\left([-1,0], R^{3}\right)$ as

$$
\dot{x}(t)=L_{\mu}\left(x_{t}\right)+f\left(\mu, x_{t}\right),
$$

where $x(t)=\left(x_{1}(t), x_{2}(t), x_{3}(t)\right)^{T} \in R^{3}$, and $L_{\mu}: C \rightarrow R, f: R \times C \rightarrow R$ are given, respectively, by

$$
\begin{gathered}
L_{\mu}(\phi)=\left(\tau_{k}+\mu\right)\left(\begin{array}{ccc}
-a & -a & 0 \\
-a z_{*} & K-1 & -a x_{*} \\
a y_{*} & a x_{*} & 0
\end{array}\right)\left(\begin{array}{l}
\phi_{1}(0) \\
\phi_{2}(0) \\
\phi_{3}(0)
\end{array}\right)+\left(\tau_{k}+\mu\right)\left(\begin{array}{ccc}
0 & 0 & 0 \\
0 & -K & 0 \\
0 & 0 & 0
\end{array}\right)\left(\begin{array}{c}
\phi_{1}(-1) \\
\phi_{2}(-1) \\
\phi_{3}(-1)
\end{array}\right), \\
f(\mu, \phi)=\left(\tau_{k}+\mu\right)\left(\begin{array}{c}
0 \\
-a \phi_{1}(0) \phi_{3}(0) \\
a \phi_{1}(0) \phi_{2}(0)
\end{array}\right) .
\end{gathered}
$$

By the Riesz representation theorem, there exists a function $\eta(\theta, \mu)$ of bounded variation for $\theta \in[-1,0]$, such that

$$
L_{\mu} \phi=\int_{-1}^{0} d \eta(\theta, 0) \phi(\theta)
$$

for $\phi \in C[-1,0]$.

In fact, we can choose

$$
\eta(\theta, \mu)=\left(\tau_{k}+\mu\right)\left(\begin{array}{ccc}
-a & -a & 0 \\
-a z_{*} & K-1 & -a x_{*} \\
a y_{*} & a x_{*} & 0
\end{array}\right) \delta(\theta)-\left(\tau_{k}+\mu\right)\left(\begin{array}{ccc}
0 & 0 & 0 \\
0 & -K & 0 \\
0 & 0 & 0
\end{array}\right) \delta(\theta+1)
$$


where $\delta$ is the Dirac delta function. For $\phi \in C^{1}\left([-1,0],\left(R^{3}\right)^{*}\right)$, define

$$
\begin{gathered}
A(\mu) \phi= \begin{cases}\frac{d \phi(\theta)}{d \theta}, & \theta \in[-1,0), \\
\int_{-1}^{0} d \eta(\mu, s) \phi(s), & \theta=0,\end{cases} \\
R(\mu) \phi= \begin{cases}0, & \theta \in[-1,0), \\
f(\mu, \phi), & \theta=0 .\end{cases}
\end{gathered}
$$

Then system (3.1) is equivalent to

$$
\dot{x}_{t}=A(\mu) x_{t}+R(\mu) x_{t}
$$

where $x_{t}(\theta)=x(t+\theta)$ for $\theta \in[-1,0]$.

For $\psi \in C^{1}\left([0,1], R^{3}\right)$, define

$$
A^{*} \psi(s)= \begin{cases}-\frac{d \psi(s)}{d s}, & s \in(0,1], \\ \int_{-1}^{0} d \eta^{T}(t, 0) \psi(-t), & s=0,\end{cases}
$$

and a bilinear inner product

$$
\langle\psi(s), \phi(\theta)\rangle=\bar{\psi}(0) \cdot \phi(0)-\int_{\theta=-1}^{0} \int_{\xi=0}^{\theta} \bar{\psi}^{T}(\xi-\theta) d \eta(\theta) \phi(\xi) d \xi,
$$

where $\eta(\theta)=\eta(\theta, 0)$. Then $A=A(0)$ and $A^{*}=A^{*}(0)$ are adjoins operators.

By the discussion in Section 2, we know that $\pm i \omega_{k} \tau_{k}$ are eigenvalues of $A$, thus they are also eigenvalues of $A^{*}$.

By direct computation, we obtain that $q(\theta)=q_{0} e^{i \theta \omega_{k} \tau_{k}}$, with

$$
q(0)=(1, \alpha, \beta)^{T}=\left(1,-\frac{a+i \omega_{k}}{a}, \frac{a\left(y_{*}-x_{*}\right)-i \omega_{k} x_{*}}{i \omega_{k}}\right)^{T},
$$

is the eigenvector of $A$ corresponding to $i \omega_{k} \tau_{k}$, and $q^{*}(s)=D q_{0}^{*} e^{i s \omega_{k} \tau_{k}}$, with

$$
q_{0}^{*}=\left(1, \alpha^{*}, \beta^{*}\right)^{T}=\left(1, \frac{i \omega_{k}\left(a-i \omega_{k}\right)}{a^{2} x_{*} y_{*}-i \omega_{k} a z_{*}}, \frac{\left(a-i \omega_{k}\right) a x_{*}}{a^{2} x_{*} y_{*}-i \omega_{k} a z_{*}}\right)^{T}
$$

is the eigenvector of $A^{*}$ corresponding to $-i \omega_{k} \tau_{k}$, where

$$
D=\frac{1}{1+\bar{\alpha} \alpha^{*}+\bar{\beta} \beta^{*}-K \tau_{k} \bar{\alpha} \alpha^{*} e^{i \omega_{k} \tau_{k}}} .
$$


Using the same notation as in [14], we compute the coordinates to describe the center manifold $C_{0}$ at $\mu=0$. Let $x_{t}$ be the solution of (3.1) when $\mu=0$. Define

$$
z(t)=\left\langle q^{*}, x_{t}\right\rangle, \quad W(t, \theta)=x_{t}(\theta)-2 \operatorname{Re}\{z(t) q(\theta)\}
$$

On the center manifold $C_{0}$, we have

$$
W(t, \theta)=W(z(t), \bar{z}(t), \theta)
$$

where

$$
W(z, \bar{z}, \theta)=W_{20}(\theta) \frac{z^{2}}{2}+W_{11}(\theta) z \bar{z}+W_{02}(\theta) \frac{\bar{z}^{2}}{2}+W_{30}(\theta) \frac{z^{3}}{6}+\cdots
$$

$z$ and $\bar{z}$ are local coordinates for center manifold $C_{0}$ in the direction of $q^{*}$ and $\bar{q}^{*}$. Note that $W$ is real if $x_{t}$ is real. We consider only real solutions. For the solution $x_{t} \in C_{0}$ of (3.1), since $\mu=0$, we have

$$
\begin{aligned}
\dot{z}(t) & =i \tau_{k} \omega_{k} z+\bar{q}^{*}(\theta) f(0, W(z, \bar{z}, \theta)+2 \operatorname{Re}\{z q(\theta)\}) \\
& =i \tau_{k} \omega_{k} z+\bar{q}^{*}(0) f(0, W(z, \bar{z}, 0)+2 \operatorname{Re}\{z q(0)\}) \stackrel{\text { def }}{=} i \tau_{k} \omega_{k} z+\bar{q}^{*}(0) f_{0}(z, \bar{z}) .
\end{aligned}
$$

We rewrite this equation as

$$
\dot{z}(t)=i \tau_{k} \omega_{k} z(t)+g(z, \bar{z})
$$

where

$$
g(z, \bar{z})=\bar{q}^{*}(0) f_{0}(z, \bar{z})=g_{20} \frac{z^{2}}{2}+g_{11} z \bar{z}+g_{02} \frac{\bar{z}^{2}}{2}+g_{21} \frac{z^{2} \bar{z}}{2}+\cdots
$$

Noticing

$$
\begin{gathered}
x_{t}(\theta)=\left(x_{1 t}(\theta), x_{2 t}(\theta), x_{3 t}(\theta)\right)=W(t, \theta)+z q(\theta)+\overline{z q}(\theta), \\
q(\theta)=(1, \alpha, \beta)^{T} e^{i \theta \omega_{k} \tau_{k},}
\end{gathered}
$$

we have

$$
\begin{aligned}
& x_{1 t}(0)=z+\bar{z}+W_{20}^{(1)}(0) \frac{z^{2}}{2}+W_{11}^{(1)}(0) z \bar{z}+W_{02}^{(1)}(0) \frac{\bar{z}^{2}}{2}+O\left(|(z, \bar{z})|^{3}\right), \\
& x_{2 t}(0)=\alpha z+\overline{\alpha z}+W_{20}^{(2)}(0) \frac{z^{2}}{2}+W_{11}^{(2)}(0) z \bar{z}+W_{02}^{(2)}(0) \frac{\bar{z}^{2}}{2}+O\left(|(z, \bar{z})|^{3}\right), \\
& x_{3 t}(0)=\beta z+\overline{\beta z}+W_{20}^{(3)}(0) \frac{z^{2}}{2}+W_{11}^{(3)}(0) z \bar{z}+W_{02}^{(3)}(0) \frac{\bar{z}^{2}}{2}+O\left(|(z, \bar{z})|^{3}\right) .
\end{aligned}
$$


Thus, form (3.17), we have

$$
\begin{aligned}
g(z, \bar{z})= & \bar{q}^{*}(0) f_{0}(z, \bar{z})=\bar{D} \tau_{k}\left(1, \overline{\alpha^{*}}, \overline{\beta^{*}}\right)\left(\begin{array}{c}
0 \\
-a x_{1 t}(0) x_{3 t}(0) \\
a x_{1 t}(0) x_{2 t}(0)
\end{array}\right) \\
= & -a \bar{D} \tau_{k} \overline{\alpha^{*}}\left[z+\bar{z}+W_{20}^{(1)}(0) \frac{z^{2}}{2}+W_{11}^{(1)}(0) z \bar{z}+W_{02}^{(1)}(0) \frac{\bar{z}^{2}}{2}+O\left(|(z, \bar{z})|^{3}\right)\right] \\
& {\left[\beta z+\overline{\beta z}+W_{20}^{(3)}(0) \frac{z^{2}}{2}+W_{11}^{(3)}(0) z \bar{z}+W_{02}^{(3)}(0) \frac{\bar{z}^{2}}{2}+O\left(|(z, \bar{z})|^{3}\right)\right] } \\
& +a \bar{D} \tau_{k} \overline{\beta^{*}}\left[z+\bar{z}+W_{20}^{(1)}(0) \frac{z^{2}}{2}+W_{11}^{(1)}(0) z \bar{z}+W_{02}^{(1)}(0) \frac{\bar{z}^{2}}{2}+O\left(|(z, \bar{z})|^{3}\right)\right] \\
& {\left[\alpha z+\overline{\alpha z}+W_{20}^{(2)}(0) \frac{z^{2}}{2}+W_{11}^{(2)}(0) z \bar{z}+W_{02}^{(2)}(0) \frac{\bar{z}^{2}}{2}+O\left(|(z, \bar{z})|^{3}\right)\right] . }
\end{aligned}
$$

Comparing the coefficients of (3.17), we get

$$
\begin{aligned}
g_{20}= & -2 a \bar{D} \tau_{k}\left(\overline{\beta^{*}} \alpha-\overline{\alpha^{*}} \beta\right), \\
g_{11}= & 2 a \bar{D} \tau_{k}\left(\overline{\beta^{*}} \operatorname{Re}(\alpha)-\overline{\alpha^{*}} \operatorname{Re}(\beta)\right), \\
g_{02}= & -2 a \bar{D} \tau_{k}\left(\overline{\beta^{*}} \bar{\alpha}-\overline{\alpha^{*}} \bar{\beta}\right), \\
g_{21}= & -a \bar{D} \tau_{k} \overline{\alpha^{*}}\left[2 W_{11}^{(3)}(0)+W_{20}^{(3)}(0)+2 \beta W_{11}^{(1)}(0)+\bar{\beta} W_{20}^{(1)}(0)\right] \\
& +a \bar{D} \tau_{k} \overline{\beta^{*}}\left[2 W_{11}^{(2)}(0)+W_{20}^{(2)}(0)+2 \alpha W_{11}^{(1)}(0)+\bar{\alpha} W_{20}^{(1)}(0)\right] .
\end{aligned}
$$

Since there are $W_{20}(\theta)$ and $W_{11}(\theta)$ in $g_{21}$, we need to compute them.

From (3.6) and (3.12), we have

$$
\begin{aligned}
\dot{W} & =\dot{x}_{t}-\dot{z} q-\dot{\overline{z q}}= \begin{cases}A W-2 \operatorname{Re}\left\{\bar{q}^{*}(0) f_{0} q(\theta)\right\}, & \theta \in[-1,0), \\
A W-2 \operatorname{Re}\left\{\bar{q}^{*}(0) f_{0} q(0)\right\}+f_{0}, & \theta=0 .\end{cases} \\
& \stackrel{\text { def }}{=} A W+H(z, \bar{z}, \theta),
\end{aligned}
$$

where

$$
H(z, \bar{z}, \theta)=H_{20}(\theta) \frac{z^{2}}{2}+H_{11}(\theta) z \bar{z}+H_{02}(\theta) \frac{\bar{z}^{2}}{2}+\cdots
$$

Expanding the above series and comparing the corresponding coefficients, we obtain

$$
\left(A-2 i \tau_{k} \omega_{k}\right) W_{20}(\theta)=-H_{20}(\theta), \quad A W_{11}(\theta)=-H_{11}(\theta), \ldots
$$


From (3.22), we know that for $\theta \in[-1,0)$,

$$
H(z, \bar{z}, \theta)=-\bar{q}^{*}(0) f_{0} q(\theta)-q^{*}(0) \bar{f}_{0} \bar{q}(0)=-g q(\theta)+\overline{g q}(\theta) .
$$

Comparing the coefficients with (3.23) gives that

$$
\begin{aligned}
& H_{20}(\theta)=-g_{20} q(\theta)-\bar{g}_{02} \bar{q}(\theta), \\
& H_{11}(\theta)=-g_{11} q(\theta)-\bar{g}_{11} \bar{q}(\theta) .
\end{aligned}
$$

From (3.24), (3.26) and the definition of $A$, it follows that

$$
\dot{W}_{20}(\theta)=2 i \tau_{k} \omega_{k} W_{20}(\theta)+g_{20} q(\theta)+\bar{g}_{02} \bar{q}(\theta) .
$$

Notice that $q(\theta)=(1, \alpha, \beta)^{T} e^{i \theta \omega_{k} \tau_{k}}$, hence

$$
W_{20}(\theta)=-\frac{i g_{20}}{\omega_{k} \tau_{k}} q(0) e^{i \theta \omega_{k} \tau_{k}}+\frac{i \bar{g}_{02}}{3 \omega_{k} \tau_{k}} \bar{q}(0) e^{-i \theta \omega_{k} \tau_{k}}+E_{1} e^{2 i \theta \omega_{k} \tau_{k}}
$$

where $E_{1}=\left(E_{1}^{(1)}, E_{1}^{(2)}, E_{1}^{(3)}\right)^{T} \in R^{3}$ is a constant vector.

Similarly, from (3.24) and (3.27), we can obtain

$$
W_{11}(\theta)=-\frac{i g_{11}}{\omega_{k} \tau_{k}} q(0) e^{i \theta \omega_{k} \tau_{k}}+\frac{i \bar{g}_{11}}{\omega_{k} \tau_{k}} \bar{q}(0) e^{-i \theta \omega_{k} \tau_{k}}+E_{2}
$$

where $E_{2}=\left(E_{2}^{(1)}, E_{2}^{(2)}, E_{2}^{(3)}\right)^{T} \in R^{3}$ is also a constant vector.

In what follows, we shall seek appropriate $E_{1}$ and $E_{2}$. From the definition of $A$ and (3.24), we obtain

$$
\begin{gathered}
\int_{-1}^{0} d \eta(\theta) W_{20}(\theta)=2 i \omega_{k} \tau_{k} W_{20}(0)-H_{20}(0) \\
\int_{-1}^{0} d \eta(\theta) W_{11}(\theta)=-H_{11}(0)
\end{gathered}
$$

where $\eta(\theta)=\eta(\theta, 0)$. By (3.22), we have

$$
\begin{gathered}
H_{20}(0)=-g_{20} q(0)-\bar{g}_{02} \bar{q}(0)+2 \tau_{k}\left(\begin{array}{c}
0 \\
-\beta \\
\alpha
\end{array}\right), \\
H_{11}(0)=-g_{11} q(0)-\bar{g}_{11} \bar{q}(0)+2 \tau_{k}\left(\begin{array}{c}
0 \\
-\operatorname{Re}(\beta) \\
\operatorname{Re}(\alpha)
\end{array}\right) .
\end{gathered}
$$


Journal of Applied Mathematics

Substituting (3.29) and (3.33) into (3.31), we obtain

$$
\left(2 i \omega_{k} \tau_{k} I-\int_{-1}^{0} e^{2 i \theta \omega_{k} \tau_{k}} d \eta(\theta)\right) E_{1}=2 \tau_{k}\left(\begin{array}{c}
0 \\
-\beta \\
\alpha
\end{array}\right)
$$

which leads to

$$
\begin{aligned}
& \left(\begin{array}{ccc}
2 i \omega_{k}+a & a & 0 \\
a z_{*} & 2 i \omega_{k}-K+1+K e^{-2 \omega_{k} \tau_{k}} & a x_{*} \\
-a y_{*} & -a x_{*} & 2 i \omega_{k}
\end{array}\right) E_{1}=2\left(\begin{array}{c}
0 \\
-\beta \\
\alpha
\end{array}\right), \\
& E_{1}^{(1)}=\frac{2 a\left(2 i \omega_{k} \beta+a \alpha x_{*}\right)}{A}, \\
& E_{1}^{(2)}=\frac{-2\left(2 i \omega_{k}+a\right)\left(2 i \omega_{k} \beta+a \alpha x_{*}\right)}{A}, \\
& E_{1}^{(3)}=\frac{2}{A} A_{1},
\end{aligned}
$$

where

$$
\begin{gathered}
A=\operatorname{det}\left(\begin{array}{ccc}
2 i \omega_{k}+a & a & 0 \\
a z_{*} & 2 i \omega_{k}-K+1+K e^{-2 \omega_{k} \tau_{k}} & a x_{*} \\
-a y_{*} & -a x_{*} & 2 i \omega_{k}
\end{array}\right), \\
A_{1}=\operatorname{det}\left(\begin{array}{ccc}
2 i \omega_{k}+a & a & 0 \\
a z_{*} & 2 i \omega_{k}-K+1+K e^{-2 \omega_{k} \tau_{k}} & -\beta \\
-a y_{*} & -a x_{*} & \alpha
\end{array}\right) .
\end{gathered}
$$

Similarly, substituting (3.30) and (3.34) into (3.32), we can get

$$
\begin{gathered}
\left(\begin{array}{ccc}
a & a & 0 \\
a z_{*} & 1 & a x_{*} \\
-a y_{*} & -a x_{*} & 0
\end{array}\right) E_{2}=2\left(\begin{array}{c}
0 \\
-\operatorname{Re}(\beta) \\
\operatorname{Re}(\alpha)
\end{array}\right), \\
E_{2}^{(1)}=\frac{2 a^{2} x_{*} \operatorname{Re}(\alpha)}{B} \\
E_{2}^{(2)}=\frac{-2 a^{2} x_{*} \operatorname{Re}(\alpha)}{B} \\
E_{2}^{(3)}=\frac{2}{B} B_{1}
\end{gathered}
$$

where

$$
B=\operatorname{det}\left(\begin{array}{ccc}
a & a & 0 \\
a z_{*} & 1 & a x_{*} \\
-a y_{*} & -a x_{*} & 0
\end{array}\right), \quad B_{1}=\operatorname{det}\left(\begin{array}{ccc}
a & a & 0 \\
a z_{*} & 1 & -\operatorname{Re}(\beta) \\
-a y_{*} & -a x_{*} & \operatorname{Re}(\alpha)
\end{array}\right)
$$


Thus, we can determine $W_{20}(0)$ and $W_{11}(0)$ from (3.29) and (3.30). Furthermore, we can determine $g_{21}$. Therefore, each $g_{i j}$ in (3.21) is determined by the parameters and delay in (3.1). Thus, we can compute the following values:

$$
\begin{gathered}
c_{1}(0)=\frac{i}{2 \tau_{k} \omega_{k}}\left(g_{11} g_{20}-2\left|g_{11}\right|^{2}-\frac{\left|g_{02}\right|^{2}}{3}\right)+\frac{g_{21}}{2}, \\
\mu_{2}=-\frac{\operatorname{Re}\left(c_{1}(0)\right)}{\operatorname{Re}\left(\lambda^{\prime}\left(\tau_{k}\right)\right)} \\
\beta_{2}=2 \operatorname{Re}\left(c_{1}(0)\right), \\
T_{2}=-\frac{\operatorname{Im}\left(c_{1}(0)\right)+\mu_{2} \operatorname{Im}\left(\lambda^{\prime}\left(\tau_{k}\right)\right)}{\tau_{k} \omega_{k}}
\end{gathered}
$$

which determine the quantities of bifurcating periodic solutions in the center manifold at the critical value $\tau_{k}$, that is, $\mu_{2}$ determines the directions of the Hopf bifurcation: if $\mu_{2}>0\left(\mu_{2}<0\right)$ then the Hopf bifurcation is supercritical (subcritical) and the bifurcating periodic solutions exist for $\tau>\tau_{k}\left(\tau<\tau_{k}\right) ; \beta_{2}$ determines the stability of the bifurcating periodic solutions: the bifurcating periodic solutions are stable (unstable) if $\beta_{2}<0\left(\beta_{2}>0\right)$; and $T_{2}$ determines the period of the bifurcating periodic solutions: the period increases (decreases) if $T_{2}>0\left(T_{2}<0\right)$.

\section{Application to Control Chaos}

In the present section, we apply the results in the previous sections to system (1.2) for the purpose of control of chaos. From Section 2, we know that under certain conditions, a family of periodic solutions bifurcate from the steady states of system (1.2) at some critical values of $\tau$ and the stability of the steady state maybe change along with increase of $\tau$. If the bifurcating periodic solution is orbitally asymptotically stable or some steady state becomes local stable, then chaos may vanish. Following this ideal, we consider the following delayed feedback control system:

$$
\begin{gathered}
\dot{x}(t)=-1.69(x(t)+y(t)), \\
\dot{y}(t)=-y(t)-1.69 x(t) z(t)+K(y(t)-y(t-\tau)), \\
\dot{z}(t)=4+1.69 x(t) y(t),
\end{gathered}
$$

which has two steady states $S_{+} \dot{\doteq}(0.65,-0.65,0.5917), S_{-} \dot{\doteq}(-0.65,0.65,0.5917)$. Clearly, when $\tau=0$ or $K=0$, system (3.1) is chaotic (as depicted in Figure 1).

For the steady state $S_{+}$or $S_{-}$, we have the corresponding characteristic equation of system (4.1) as follows:

$$
\lambda^{3}+(2.69-K) \lambda^{2}+(6.76-1.69 K) \lambda+22.8488+K\left(\lambda^{2}+1.69 \lambda\right) e^{-\lambda \tau}=0 .
$$

Clearly, when $\tau=0,(4.2)$ has a negative root and a pair of complex roots with positive real parts. Following Section 2, we can obtain $p=-2 K-6.2839, q=22.8488 K-77.2289$, $r=522.0677>0, \Delta=p^{2}-3 q>0$, and $z_{1}^{*}=(1 / 3)(-p+\sqrt{\Delta})>0$ for all $K \in R$. When 


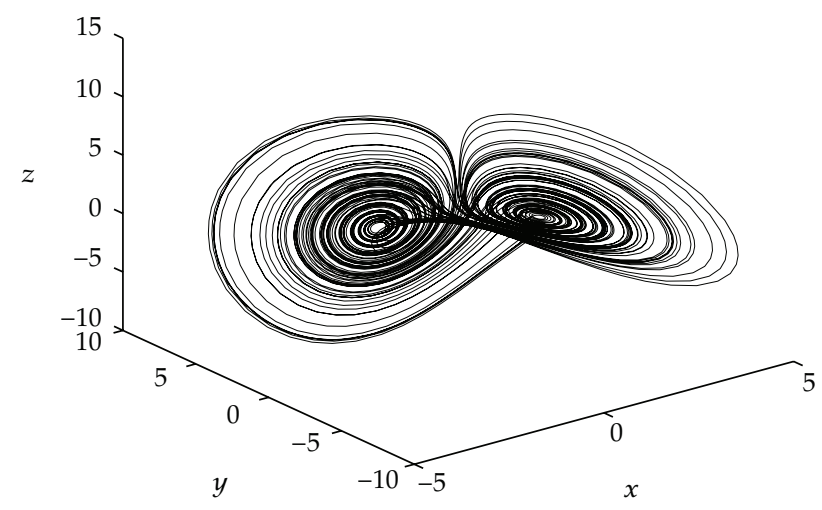

Figure 2: Chaos still exists for $K=-1, \tau=0.2$.

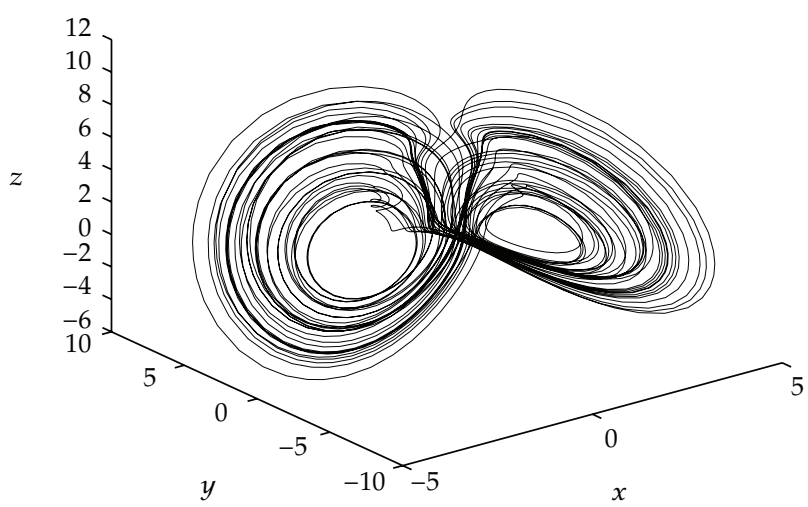

Figure 3: Chaos still exists for $K=-1, \tau=2.5$.

$K<-0.1907$ or $K>12.107, h\left(z^{*}\right)<0$. Thus, from Lemma 2.6 and Theorem 2.9 , we know that (4.2) has roots with positive real parts. In particular, we have $K=-1$, that is,

$$
\begin{gathered}
\dot{x}(t)=-1.69(x(t)+y(t)), \\
\dot{y}(t)=-y(t)-1.69 x(t) z(t)-(y(t)-y(t-\tau)), \\
\dot{z}(t)=4+1.69 x(t) y(t) .
\end{gathered}
$$

In this case, we can compute

$$
\begin{aligned}
& p \doteq-4.2839, \quad q \doteq-100.077, \quad r \dot{=} 522.0677, \quad \Delta \doteq 318.5850, \\
& z_{1} \doteq 8.9478, \quad \omega_{1}=2.9913, \quad \tau_{1}^{(j)} \dot{=} 0.2205+\frac{2 j \pi}{\omega_{1}}, \quad h^{\prime}\left(z_{1}\right) \dot{=} 63.4487, \\
& z_{2} \dot{=} 5.6545, \quad \omega_{2}=2.3779, \quad \tau_{2}^{(j)} \doteq 0.5227+\frac{2 j \pi}{\omega_{2}}, \quad h^{\prime}\left(z_{2}\right) \doteq-52.6043 .
\end{aligned}
$$




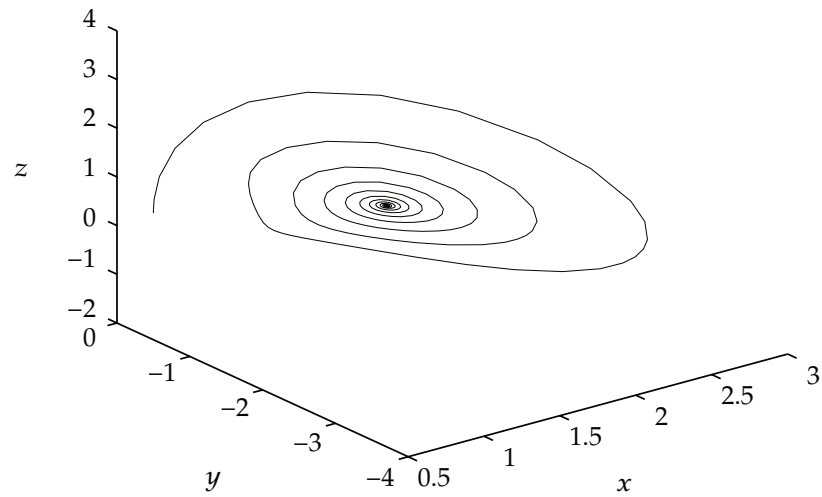

Figure 4: When $K=-1, \tau=0.8$, chaos vanishes, and $S_{1}$ becomes local stable. Here initial value is $(0.5,-0.5,0.6)$.

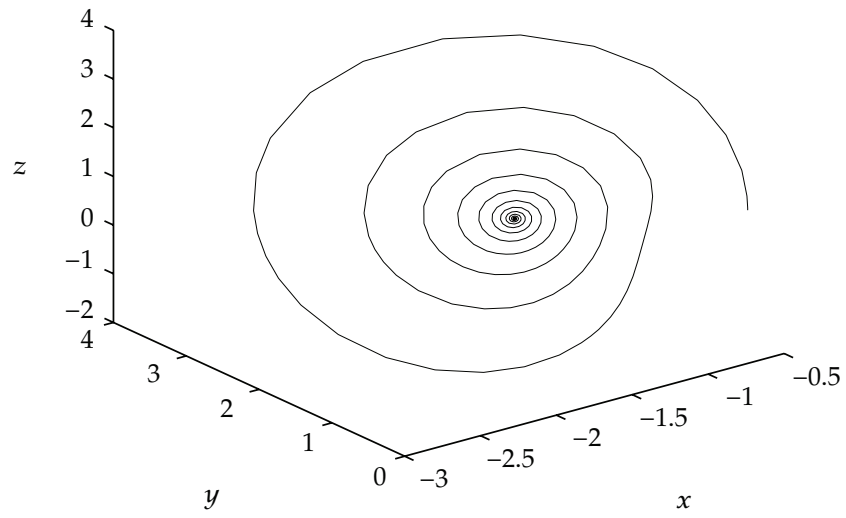

Figure 5: When $K=-1, \tau=0.8$, chaos vanishes, and $S_{2}$ becomes local stable. Here initial value is $(-0.5,0.5,0.6)$.

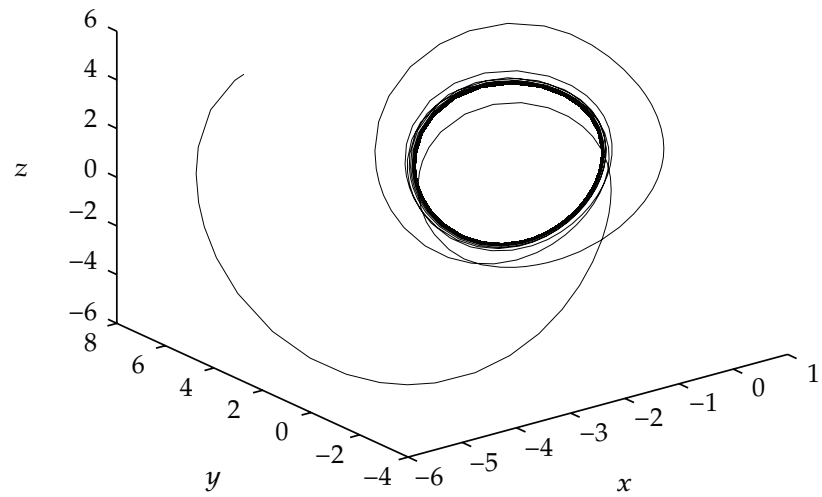

Figure 6: When $K=-1, \tau=2$, chaos vanishes, and $S_{2}$ becomes a stable periodic solution. Here initial value is $(5,-5,5)$. 

notice that

Thus, from Lemma 2.7, we have $\operatorname{Re} \lambda\left(\tau_{1}^{(j)}\right) / d \tau>0$ and $\operatorname{Re} \lambda\left(\tau_{2}^{(j)}\right) / d \tau<0$. In addition,

$$
\tau_{1}^{(0)} \dot{=} 0.2205<\tau_{2}^{(0)} \dot{=} 0.5227<\tau_{1}^{(1)} \dot{=} 2.3210<\tau_{2}^{(1)} \dot{=} 3.1650
$$

Thus, from Theorem 2.8, we have the following conclusion about the stability of the steady states of system (4.3) and Hopf bifurcation.

\section{Conclusion}

Suppose that $\tau_{k}^{(j)}, k=1,2 ; j=0,1,2, \ldots$ is defined by (4.4).

(i) When $\tau \in\left[0, \tau_{2}^{(0)}\right) \cup\left(\tau_{1}^{(1)}, \infty\right)$, the steady states $S_{1}$ and $S_{2}$ of the system (4.1) are unstable (see Figures 2 and 3 ).

(ii) When $\tau \in\left(\tau_{2}^{(0)}, \tau_{1}^{(1)}\right)$, the steady states $S_{1}$ and $S_{2}$ of the system (4.1) are asymptotically (see Figures 4 and 5).

(iii) When $\tau=\tau_{k}^{(j)}$, system (4.1) undergoes a Hopf bifurcation at the steady states states $S_{1}$ and $S_{2}$.

The above simulations indicate that when the steady state is stable or the bifurcating periodic solutions are orbitally asymptotically stable, chaos vanishes (see Figures 4-6).

\section{References}

[1] J. H. Ma and Y. S. Chen, "Study for the bifurcation topological structure and the global complicated character of a kind of nonlinear finance system. I," Applied Mathematics and Mechanics, vol. 22, no. 11, pp. 1240-1251, 2001.

[2] J. H. Ma and Y. S. Chen, "Study for the bifurcation topological structure and the global complicated character of a kind of nonlinear finance system. II," Applied Mathematics and Mechanics, vol. 22, no. 12, pp. 1375-1382, 2001.

[3] J. H. Ma, B. Ren, and Y. S. Chen, "Impulsive control of chaotic attractors in nonlinear chaotic systems," Applied Mathematics and Mechanics, vol. 25, no. 9, pp. 889-894, 2004.

[4] J. Ding, W. Yang, and H. Yao, "A new modified hyperchaotic finance system and its control," International Journal of Nonlinear Science, vol. 8, no. 1, pp. 59-66, 2009.

[5] W.-C. Chen, "Dynamics and control of a financial system with time-delayed feedbacks," Chaos, Solitons and Fractals, vol. 37, no. 4, pp. 1198-1207, 2008.

[6] Q. Gao and J. Ma, "Chaos and Hopf bifurcation of a finance system," Nonlinear Dynamics, vol. 58, no. 1-2, pp. 209-216, 2009.

[7] Y. Wang, Y. H. Zhai, and J. Wang, "Chaos and Hopf bifurcation of a finance system with distributed time delay," International Journal of Applied Mathematics and Mechanics, vol. 6, pp. 1-13, 2010.

[8] W.-S. Son and Y.-J. Park, "Delayed feedback on the dynamical model of a financial system," Chaos, Solitons \& Fractals, vol. 44, no. 4-5, pp. 208-217, 2011.

[9] E. Ott, C. Grebogi, and J. A. Yorke, "Controlling chaos," Physical Review Letters, vol. 64, no. 11, pp. 1196-1199, 1990.

[10] K. Pyragas, "Continuous control of chaos by self-controlling feedback," Physics Letters A, vol. 170, no. 6, pp. 421-428, 1992.

[11] S. Wiggins, Introduction to Applied Nonlinear Dynamical Systems and Chaos, vol. 2 of Texts in Applied Mathematics, Springer, New York, NY, USA, 2nd edition, 2003.

[12] S. Ruan and J. Wei, "On the zeros of transcendental functions with applications to stability of delay differential equations with two delays," Dynamics of Continuous, Discrete \& Impulsive Systems A, vol. 10, no. 6, pp. 863-874, 2003. 
[13] Y. Song and J. Wei, "Bifurcation analysis for Chen's system with delayed feedback and its application to control of chaos," Chaos, Solitons and Fractals, vol. 22, no. 1, pp. 75-91, 2004.

[14] B. D. Hassard, N. D. Kazarinoff, and Y. H. Wan, Theory and Applications of Hopf Bifurcation, vol. 41 of London Mathematical Society Lecture Note Series, Cambridge University Press, Cambridge, UK, 1981. 


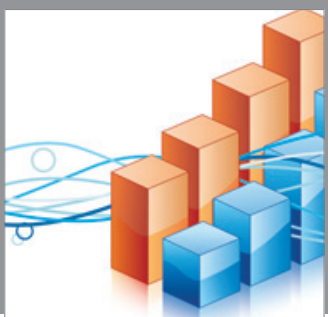

Advances in

Operations Research

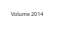

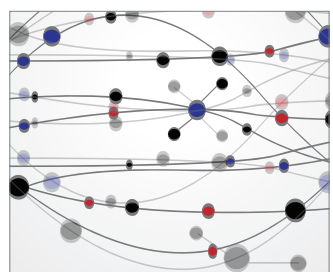

\section{The Scientific} World Journal
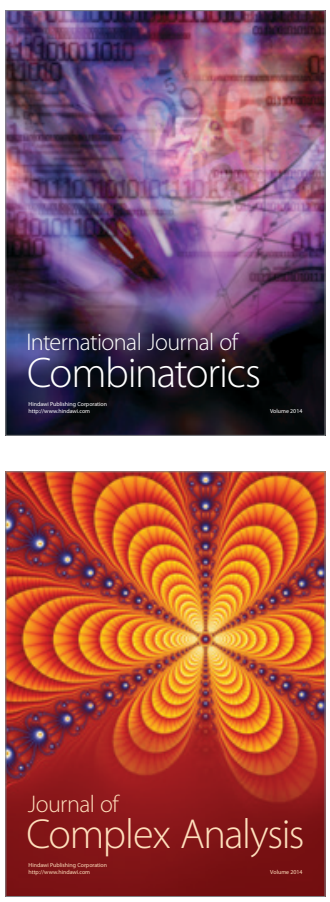

International Journal of

Mathematics and

Mathematical

Sciences
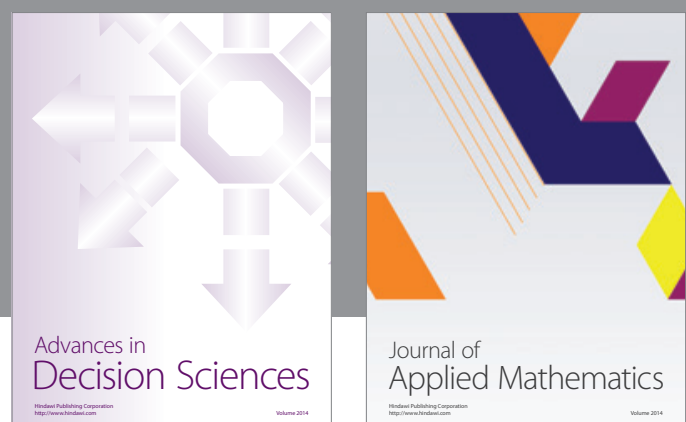

Journal of

Applied Mathematics
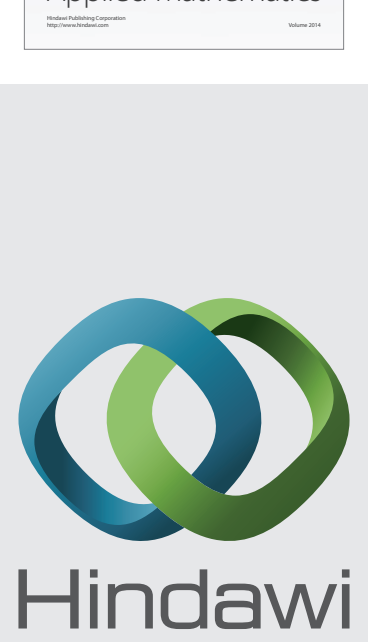

Submit your manuscripts at http://www.hindawi.com
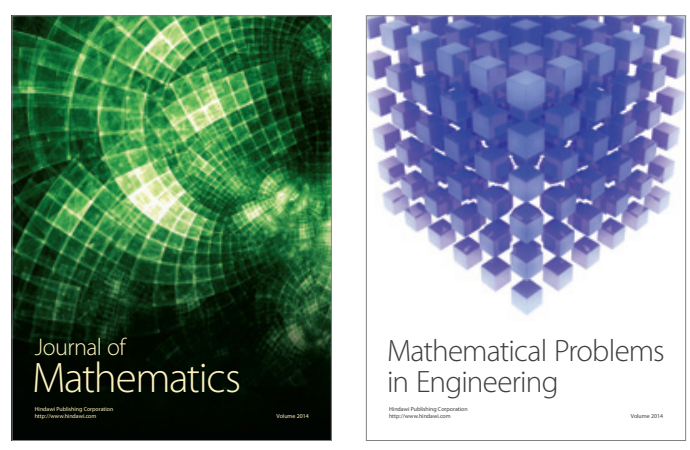

Mathematical Problems in Engineering
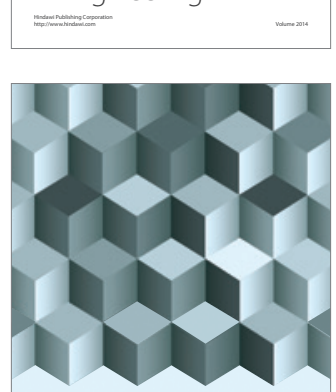

Journal of

Function Spaces
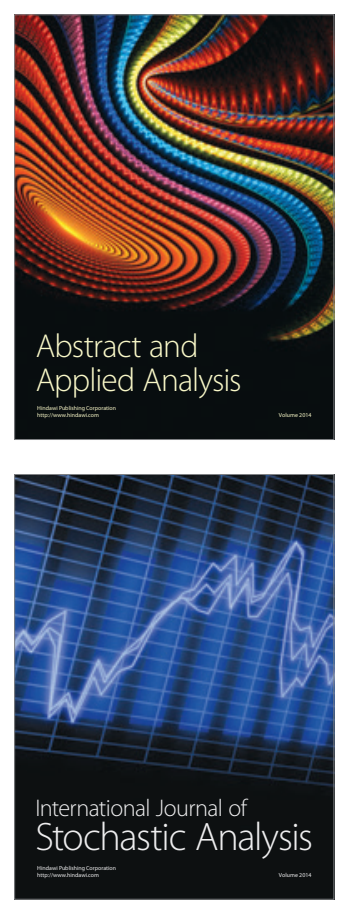

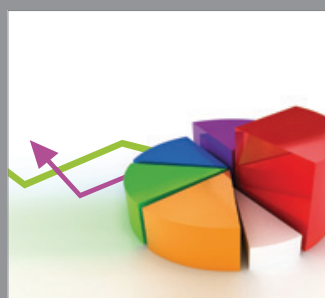

ournal of

Probability and Statistics

Promensencen
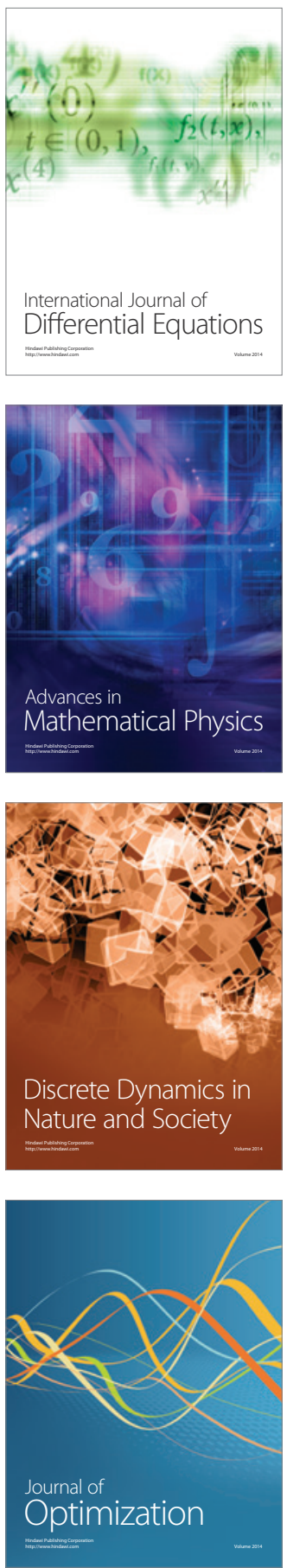